\title{
MINIMAL SURFACES WITH CONSTANT GAUSS CURVATURE
}

\author{
BANG-YEN CHEN ${ }^{1}$
}

AbstraCt. Minimal surfaces with constant Gauss curvature in real space forms are studied.

1. Introduction. Let $R^{m}(c)$ be an $m$-dimensional complete, simplyconnected Riemannian manifold of constant curvature $c$ with covariant differentiation $\nabla$, and let $M$ be a surface immersed in $R^{m}(c)$. For a normal vector field $\eta$ of $M$ in $R^{m}(c)$, we denote by $\nabla^{*} \eta$ the normal component of $\nabla \eta$. Then $\nabla^{*}$ defines a connection in the normal bundle. A normal vector field $\eta$ is said to be parallel in the normal bundle if $\nabla^{*} \eta=0$ and to be constant if $\nabla \eta=0$.

MAIN THEOREM. Let $M$ be a minimal surface with constant Gauss curvature in a space form $R^{m}(c)$ of curvature $c$. If there exists a nonconstant normal vector field which is parallel in the normal bundle, then $c>0$ and $M$ is an open piece of the Clifford torus.

Since every unit normal vector field is parallel in the normal bundle for $m=3$ and is constant only if $M$ is totally geodesic, we have the following corollary.

COROLlaRY 1. If $M$ is a minimal surface with constant Gauss curvature in a 3-space form $R^{3}(c)$, then either $M$ is totally geodesic or $c>0$ and $M$ is an open piece of the Clifford torus.

REMARK 1. Corollary 1 was obtained by Lawson [4] for $c>0$. If $c=0$, then Corollary 1 also follows from the well-known Ricci condition.

REMARK 2. Suppose $R^{m}(c)$ is the euclidean $m$-space $E^{m}$. Then by combining a result of Osserman-Chern [5] for the generalized Gauss map and a result of Calabi [1] for Riemann surfaces in the complex projective spaces, we know that every minimal surface in the euclidean $m$-space with constant Gauss curvature must be totally geodesic.

Received by the editors October 1, 1971.

AMS 1970 subject classifications. Primary 53A10, 53B25; Secondary 53C40.

Key words and phrases. Minimal surface, Gauss curvature, scalar normal curvature, Clifford torus.

${ }^{1}$ This work was partially supported by the National Science Foundation under grant GU-2648.

(c) American Mathematical Society 1972 
Remark 3. For the minimal surfaces with positive constant Gauss curvature in the $m$-sphere, see do Carmo-Wallach [2].

The author would like to express his thanks to Professor H. B. Lawson for the valuable conversations.

2. Preliminaries. Let $R^{m}(c)$ be a complete, simply-connected Riemannian manifold of constant sectional curvature $c$, with scalar product $\langle$,$\rangle , and let M$ be a surface immersed in $R^{m}(c)$. We choose a local field of orthonormal frames $e_{1}, \cdots, e_{m}$ in $R^{m}(c)$ such that, restricted to $M$, the vectors $\boldsymbol{e}_{1}, \boldsymbol{e}_{2}$ are tangent to $M$ (and, consequently, $\boldsymbol{e}_{3}, \cdots, \boldsymbol{e}_{m}$ are normal to $M$ ). With respect to the frame field chosen above, let $\omega^{1}, \cdots, \omega^{m}$ be the field of dual frame. Then the structure equations of $R^{m}(c)$ are given by

(1) $d \omega^{A}=-\sum \omega_{B}^{A} \wedge \omega^{B}, \quad \omega_{B}^{A}+\omega_{A}^{B}=0$, $A, B, C, \cdots=1, \cdots, m$.

(2) $d \omega_{B}^{A}=-\sum \omega_{C}^{A} \wedge \omega_{B 3}^{C}+c \omega^{A} \wedge \omega^{B}$,

We restrict these forms to $M$. Then $\omega^{r}=0$, for $r, s, t, \cdots=3, \cdots, m$. Since $0=d \omega^{r}=\omega_{r}^{1} \wedge \omega^{1}+\omega_{r}^{2} \wedge \omega^{2}$, by Cartan's lemma we may write

$$
\omega_{i}^{r}=\sum h_{i j}^{r} \omega^{j}, \quad h_{i j}^{r}=h_{j i}^{r}, \quad i, j=1,2 .
$$

From these we obtain

$$
\begin{aligned}
& d \omega^{i}=-\sum \omega_{j}^{i} \wedge \omega^{j}, \\
& d \omega_{2}^{1}=\left\{c+\sum_{r} \operatorname{det}\left(h_{i j}^{r}\right)\right\} \omega^{1} \wedge \omega^{2}, \\
& d \omega_{i}^{r}=-\sum \omega_{j}^{r} \wedge \omega_{i}^{j}-\sum \omega_{s}^{r} \wedge \omega_{i}^{s} .
\end{aligned}
$$

The second fundamental form $\boldsymbol{h}$, the mean curvature vector $\boldsymbol{H}$, the Gauss curvature $K$ and the scalar normal curvature $K_{N}$ are given by

$$
\begin{aligned}
\boldsymbol{h} & =\sum_{r, i, j} h_{i j}^{r} \omega^{i} \omega^{j} \boldsymbol{e}_{r}, \\
\boldsymbol{H} & =\frac{1}{2} \sum\left(h_{11}^{r}+h_{22}^{r}\right) \boldsymbol{e}_{r}, \\
K & =c+\sum_{r} \operatorname{det}\left(h_{i j}^{r}\right), \\
K_{N} & =\sum_{r, s=3}^{m}\left\{\sum_{i=1}^{2}\left(h_{1 i}^{r} h_{2 i}^{s}-h_{1 i}^{s} h_{2 i}^{r}\right)\right\}^{2},
\end{aligned}
$$

respectively. A surface $M$ is minimal if $\boldsymbol{H}=0$ identically on $M$. 
3. Proof of the Main Theorem. We first prove the following lemmas:

LEMMA 1. Let $M$ be a minimal surface of $R^{m}(c)$. If there exists a unit normal vector field $e$ which is parallel in the normal bundle, then $M=$ $M_{1} \cup M_{2}$, where $M_{1}$ is an open subset of $M$ with $K_{N}=0$ and $M_{2}=$ $\left\{p \in M:\langle\boldsymbol{h}(\boldsymbol{X}, \boldsymbol{Y}), \boldsymbol{e}\rangle=0\right.$ for all tangent vectors $\boldsymbol{X}, \boldsymbol{Y}$ in $\left.T_{p}(M)\right\}$.

Proof. Let $M_{1}=\left\{p \in M: \exists X, Y \in T_{p}(M)\right.$ such that $\left.\langle\boldsymbol{h}(\boldsymbol{X}, \boldsymbol{Y}), \boldsymbol{e}\rangle \neq 0\right\}$. Then $M_{1}$ is an open subset of $M$. We choose our frame field in such a way that

$$
e_{3}=e, \quad h_{12}^{3}=0,
$$

on $M_{1}$. Then we have

$$
h_{11}^{3}=-h_{22}^{3} \neq 0
$$

everywhere on $M_{1}$. Since $e$ is parallel in the normal bundle, we have

$$
\omega_{r}^{3}=0 \text {. }
$$

By taking exterior differentiation of (13) and applying (4), (6) and (13), we obtain

$$
h_{12}^{r} h_{11}^{3}=0, \quad \text { for } r=4, \cdots, m \text {. }
$$

From (10), (12) and (14), we see that the scalar normal curvature $K_{N}=0$ identically on $M_{1}$. This proves Lemma 1 .

LeMmA 2. If $M$ is a minimal surface of $R^{m}(c)$ with constant Gauss curvature and zero scalar normal curvature, then $M$ is either flat and $c \geqq 0$ or totally geodesic.

Proof. Since the scalar normal curvature $K_{N}=0$ identically on $M$, we have $\sum_{i=1}^{2}\left(h_{1 i}^{r} h_{2 i}^{s}-h_{1 i}^{s} h_{2 i}^{r}\right)=0$ for $r, s=3, \cdots, m$. Thus, we may choose our local frame field in such a way that

$$
h_{12}^{r}=0, \quad r=3, \cdots, m .
$$

Therefore we obtain

$$
\omega_{1}^{r}=h_{11}^{r} \omega^{1}, \quad \omega_{2}^{r}=-h_{11}^{r} \omega^{2} .
$$

Taking exterior differentiation of the first equation of (16), we have

$$
d h_{11}^{r} \wedge \omega^{1}+2 h_{11}^{r} d \omega^{1}=\sum h_{11}^{s} \omega^{1} \wedge \omega_{s}^{r} .
$$

Multiplying (17) by $h_{11}^{r}$ and summing up on $r$, we obtain

$$
\sum\left(h_{11}^{r} d h_{11}^{r}\right) \wedge \omega^{1}+2 \sum\left(h_{11}^{r}\right)^{2} d \omega^{1}=\sum\left(h_{11}^{r} h_{11}^{s}\right) \omega^{1} \wedge \omega_{s}^{r} .
$$


Since $\omega_{s}^{r}=-\omega_{r}^{s}$, this equation implies

$$
\sum\left(h_{11}^{r} d h_{11}^{r}\right) \wedge \omega^{1}+2 \sum\left(h_{11}^{r}\right)^{2} d \omega^{1}=0 .
$$

On the other hand, the constancy of the Gauss curvature implies

$$
\sum h_{11}^{r} d h_{11}^{r}=0 \text {. }
$$

Therefore, combining (18) and (19), we obtain

$$
\sum\left(h_{11}^{r}\right)^{2} d \omega^{1}=0 .
$$

Similarly, by taking exterior differentiation of the second equation of (16), we obtain

$$
\sum\left(h_{11}^{r}\right)^{2} d \omega^{2}=0
$$

Put $V=\left\{p \in M: d \omega^{1} \neq 0\right.$ or $\left.d \omega^{2} \neq 0\right\}$. Then $V$ is an open subset of $\boldsymbol{M}$. If $V=\varnothing$, then $d \omega^{1}=d \omega^{2}=0$ identically on $M$. Hence, by (4), (5) and (9), we see that $K=0$ on $M$. Now, suppose that $V \neq \varnothing$, and $V_{1}$ is a component of $V$. Then we have

$$
h_{11}^{r}=0, \quad r=3, \cdots, m,
$$

on $V_{1}$. This implies that $V_{1}$ is totally geodesic in $R^{m}(c)$. If $c=0$, then $K=0$ identically on $M$. Now assume that $c \neq 0$. Then $V_{1}$ is of constant curvature $c$. This implies that closure $(V)=M$. Thus $M$ is totally geodesic. This proves Lemma 2.

LEMMA 3. Let $M$ be a surface in a 3-dimensional totally geodesic submanifold $R^{3}(c)$ of $R^{m}(c)$ with unit normal vector $\bar{e}$ in $R^{3}(c)$. If $e$ is a unit normal vector field of $M$ in $R^{m}(c)$ which is parallel in the normal bundle, then there exist a constant unit normal vector field $a$ in $R^{m}(c)$ and $a$ constant angle $\alpha$ such that $\boldsymbol{e}=(\cos \alpha) \overline{\boldsymbol{e}}+(\sin \alpha) \boldsymbol{a}$.

This lemma can be proved immediately.

Now, we return to the proof of the Main Theorem. Let $M$ be a minimal surface of a space form $R^{m}(c)$ with constant Gauss curvature $K$. If there exists a nonconstant unit normal vector field $e$ which is parallel in the normal bundle, then by Lemma 1 we see that $M=M_{1} \cup M_{2}$, where $M_{1}$ is open with scalar normal curvature $K_{N}=0$ and $M_{2}=M-M_{1}=$ $\{p \in M:\langle\boldsymbol{h}(\boldsymbol{X}, \boldsymbol{Y}), \boldsymbol{e}\rangle=0$ for all tangent vectors $\boldsymbol{X}, \boldsymbol{Y}$ at $p\}$. Since $\boldsymbol{e}$ is nonconstant, $M_{1}$ is nonempty. For each $p \in M_{1}$, there exist tangent vectors $\boldsymbol{X}, \boldsymbol{Y}$ at $p$ such that $\langle\boldsymbol{h}(\boldsymbol{X}, \boldsymbol{Y}), \boldsymbol{e}\rangle \neq 0$. Hence by Lemma 2 we see that $\boldsymbol{M}$ is flat and not totally geodesic in $R^{m}(c)$. Therefore by (9) we obtain $c>0$, i.e., $R^{m}(c)$ is an $m$-sphere $S^{m}(c)$. Now let $U$ be a component of $M$. Then, 
by $K=K_{N}=0, U$ is an open piece of a Clifford torus in a great 3-sphere $S^{3}(c)$ [3]. Let $\bar{e}$ denote the unit normal vector field of $U$ in $S^{3}(c)$ and $e_{1}, e_{2}$ be in the principal directions of $\bar{e}$. Then we can find that the second fundamental form in the direction of $\overline{\boldsymbol{e}}$ is given by

$$
\left(\left\langle h\left(e_{i}, e_{j}\right), \bar{e}\right\rangle\right)=\left(\begin{array}{cc}
c^{1 / 2} & 0 \\
0 & -c^{1 / 2}
\end{array}\right) .
$$

Therefore by Lemma 3 we see that the second fundamental form in the direction of $e$ is given by

$$
\left(\left\langle h\left(e_{i}, e_{j}\right), e\right\rangle\right)=\left(\begin{array}{cc}
c^{1 / 2} \cos \alpha & 0 \\
0 & -c^{1 / 2} \cos \alpha
\end{array}\right)
$$

where $\cos \alpha$ is a nonzero constant. Therefore, by the continuity of the second fundamental form $h$ on $M$ and the definition of $M_{2}$, we see that $M_{1}=M$. This implies that $M$ is an open piece of a Clifford torus in a 3-sphere $S^{3}(c) \subset S^{m}(c)$. This completes the proof of the theorem.

\section{REFERENCES}

1. E. Calabi, lsometric imbedding of complex manifolds, Ann. of Math. (2) 58 (1953), 1-23. MR 15, 160.

2. M. P. do Carmo and N. R. Wallach, Minimal immersions of spheres into spheres, Ann. of Math. (2) 93 (1971), 43-62.

3. K. Kenmotsu, Some remarks on minimal submanifolds, Tôhoku Math. J. (2) 22 (1970), 240-248. MR 42 \#3688.

4. H. B. Lawson, Jr., Local rigidity theorems for minimal hypersurfaces, Ann. of Math. (2) 89 (1969), 187-197. MR 38 \#6505.

5. R. Osserman and S. S. Chern, Complete minimal surfaces in euclidean n-space, J. Analyse Math. 19 (1967), 15-34. MR 37 \#2103.

Department of Mathematics, Michigan State University, East Lansing, MichigAN 48823 